\title{
Extreme Entvölkerungsgemeinden in der Schweiz
}

\author{
Rudolf Butz
}

\section{Problemstellung}

Während die Bevölkerung in der Schweiz gesamthaft ansteigt, zeigen im ganzen Land Gemeinden mehr oder weniger starke Bevölkerungsverluste, die in zahlreichen Fällen seit 185060 und mehr Prozent betrugen. Nachfolgend soll untersucht werden, ob solche Bevölkerungsabnahmen mit besonders lange anhaltenden Verlustperioden einhergingen und wo sich Entvölkerungsgemeinden unter Berücksichtigung beider Kriterien häufen. Gleichzeitig werden für die Gemeinden mit extrem starken prozentualen Abnahmen diese für den Zeitraum von 1850 bis 1960 berechnet, da sie anläßlich der Volkszählung von 1960 nicht veröffentlicht wurden.

\section{Prozentuale Abnahme}

Tabelle 1 enthält alle Gemeinden der Schweiz, deren Bevölkerungsabnahme von 1850 bis 1960 mindestens 60 Prozent betrug. Rund zwei Drittel, nämlich 20 dieser 31 Gemeinden, befinden sich allein im Tessin, wovon neun im Bezirk Locarno und von diesen wiederum fünf im Onsernonetal. Sechs weitere Beispiele sind in der Leventina, hauptsächlich auf den Trogschultern oberhalb Faido und Lavorgo, zu finden, drei andere im Maggiatal und je eines im Bleniotal und im Mendrisiotto.

In wesentlich geringerem Maße häufen sich große Gesamtverluste im Mittelland der aneinandergrenzenden Kantone Freiburg und Waadt. Allerdings ist zu berücksichtigen, da $\beta$ dieser Raum besonders kleine Gemeinden aufweist, von ihnen gehört die kleinste Gemeinde der Schweiz, Illens, zur besprochenen Gruppe. In anderen Kantonen würde eine so kleine Siedlung kaum als Gemeinde erscheinen und deshalb auch nicht in der gemeindeweisen Statistik figurieren. Von den übrigen sieben Gemeinden mit extremen Verlusten liegen drei im Berner bzw. im Waadtländer Jura, während sich die verbleibenden auf verschiedene Gebiete verteilen.

Von den genannten Gemeinden befinden sich 21 im südlichen, weitere acht im westlichen Landesteil, einschließlich des Kantons Bern, und nur zwei im östlichen, nicht aber in der Ostschweiz im engeren Sinne. Unter ihnen sei Sternenberg als Sonderfall in einer stark industrialisierten Region und im bevölkerungsreichsten Kanton speziell erwähnt, beson- ders, weil es den größten Gesamtverlust aller Mittellandgemeinden aufweist. Immerhin sei darauf hingewiesen, da $B$ die Landschaft um Sternenberg bereits deutliche Eigenschaften des Überganges zu den Alpen aufweist.

Von insgesamt 22 in den Alpen liegenden Entvölkerungsgemeinden ist nur eine, Isenfluh, am Nordhang anzutreffen, die übrigens mit $60,0 \%$ Gesamtabnahme nur knapp zur untersuchten Kategorie fällt. Im Mittelland liegen fünf, im Jura einschließlich des Kantons Schaffhausen vier der erwähnten Gemeinden.

\section{Gleichmäßigkeit der Abnahme}

In den meisten Gemeinden der bisher betrachteten Gruppe war indessen die Abnahme keineswegs besonders lang anhaltend, vielmehr waren in fast allen Fällen vorübergehende Zunahmen, die sich in einzelnen Volkszählungen auswirkten, zu verzeichnen. Die Gemeinden der Schweiz, in denen fortlaufende Verluste in mindestens acht aufeinanderfolgenden Volkszählungen zum Ausdruck kamen, sind in Tabelle 2 zusammengestellt. Mit einbezogen wurden noch Gemeinden, deren fortlaufende Abnahme in dieser Zeit durch Stagnation oder einmalige Zunahme um höchstens eine Person unterbrochen wurde. Als Folge dieses Verfahrens erscheinen allerdings die Gemeinden Möhntal und Campo-Vallemaggia in der Aufstellung zweimal.

Abgesehen von sieben Gemeinden im Tessin handelt es sich bei den in Tabelle 2 angeführten durchwegs um andere als zuvor. Auch die Verteilung auf verschiedene Gebiete ist wesentlich anders. Der Anteil der Alpen ist geringer, während der des Mittellandes und des Jura untereinander ungefähr gleichbleibt. Auf West-, Süd- und Ostschweiz entfällt je ein Drittel der Gemeinden dieser Gruppe, auf die Ostschweiz im engeren Sinne entfallen allerdings nur drei Gemeinden.

Gleichmäßige Abnahme über acht Jahrzehnte war nicht wesentlich häufiger als über neun. Besonders oft waren ununterbrochene Abnahmen von 1880 bzw. 1870 bis 1960 festzustellen, während für den Zeitraum von 1870 bis 1950 kein Beispiel vorliegt. Drei Gemeinden zeigen anhaltende Abnahmen über 100 Jahre, dazu kommt noch eine mit einmaliger Zunahme um eine Person. 


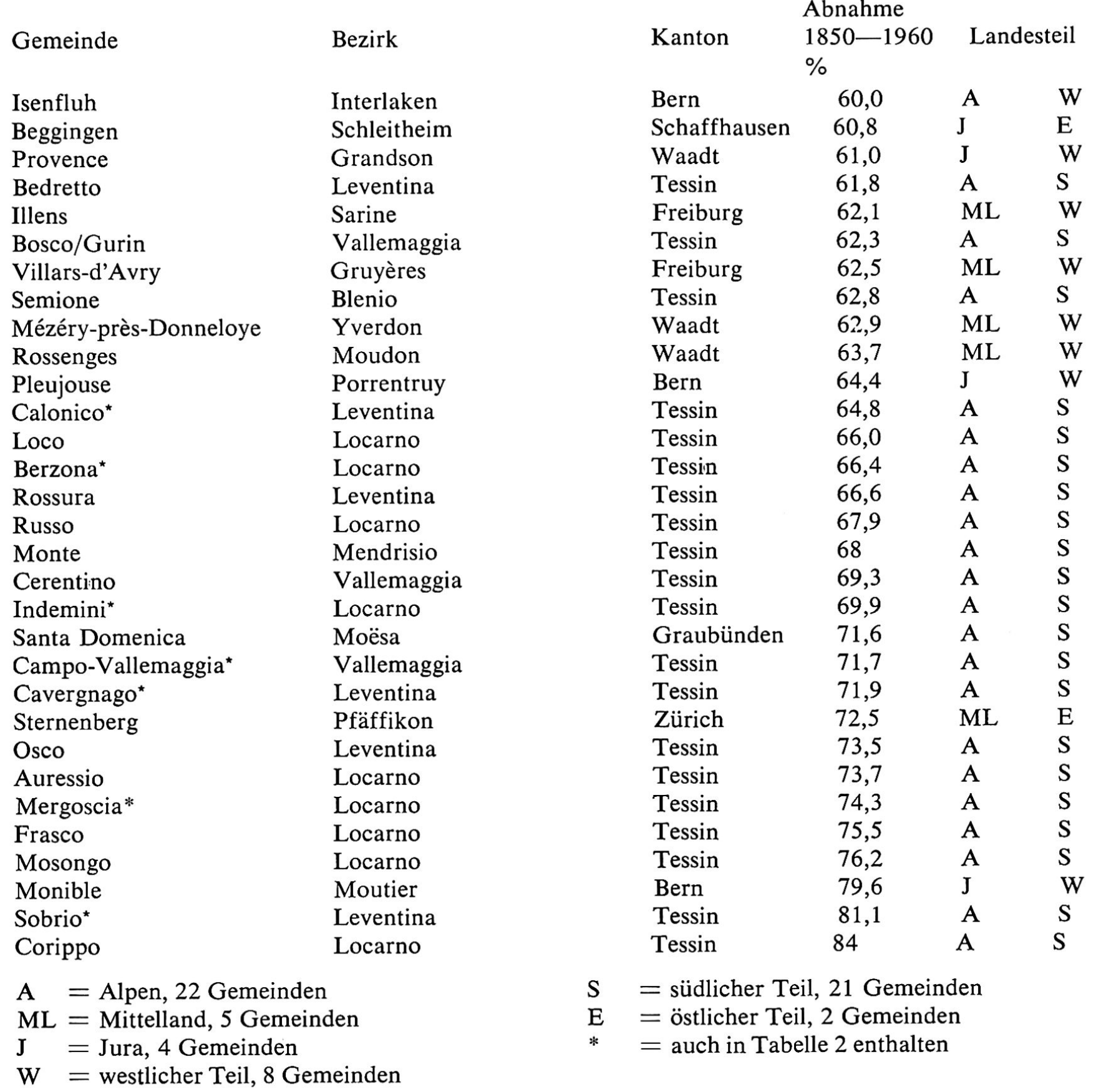

Legende zu Tabelle 2
$\mathrm{A}=$ Alpen, 21 Gemeinden
$\mathrm{ML}=$ Mittelland, 12 Gemeinden
$\mathrm{J}=$ Jura, 10 Gemeinden
$\mathrm{W}=$ westlicher Teil, 15 Gemeinden
$S=$ südlicher Teil, 14 Gemeinden

$\mathrm{E}=$ östlicher Teil, 14 Gemeinden

1 auch in Tabelle 1 enthalten

21956 mit einigen weiteren Gemeinden zu Valcolla vereinigt

3 in der Tabelle doppelt angeführt 
Tabelle 2. Gemeinden mit anhaltender Bevölkerungsabnahme

\begin{tabular}{|c|c|c|c|c|c|c|}
\hline $\begin{array}{l}\text { Zeitraum } \\
80 \text { Jahre }\end{array}$ & Gemeinde & Bezirk & \multicolumn{3}{|c|}{ anton, Landesteil } & Bemerkungen \\
\hline \multirow{6}{*}{$1850-1930$} & Kulmerau & Sursee & I U & MI & $\mathrm{F}$ & \\
\hline & Biezwil & Bucheggberg & SO & $\mathrm{ML}$ & $\mathrm{W}$ & \\
\hline & Safien & Heinzenberg, Kreis Safien & GR & A & $\mathrm{E}$ & \\
\hline & Cavagnago $^{1}$ & Leventina & $\mathrm{TI}$ & A & $S$ & \\
\hline & Bargen & Schaffhausen & $\mathrm{SH}$ & $\mathbf{J}$ & $\mathrm{E}$ & Stagnation $1850 / 1860$ \\
\hline & Nufenen & Hinterrhein, Kreis Rheinw. & GR & A & $\mathrm{E}$ & Stagnation $1920 / 1930$ \\
\hline \multirow[t]{3}{*}{$1860-1941$} & Trogen & Vorderland & AR & A & $\mathrm{E}$ & \\
\hline & Brione & Locarno & $\mathrm{TI}$ & A & $S$ & \\
\hline & Bagnes & Entremont & VS & A & W & \\
\hline $1870-1950$ & Signôra2 & Lugano & $\mathrm{TI}$ & A & S & \\
\hline \multirow{9}{*}{$1880-1960$} & Rüeggisberg & Seftigen & $\mathrm{BE}$ & $\mathrm{ML}$ & W & \\
\hline & Staffelbach & Zofingen & $\mathrm{AG}$ & ML & $\mathrm{E}$ & \\
\hline & Longirod & Aubonne & VD & $\mathbf{J}$ & W & \\
\hline & Montalchez & Boudry & $\mathrm{NE}$ & J & W & \\
\hline & Les Bayards & Val-de-Travers & $\mathrm{NE}$ & $\mathbf{J}$ & $\mathrm{W}$ & \\
\hline & Sobrio & Leventina & TI & A & $\mathrm{S}$ & Stagnation 1930/1941 \\
\hline & Tremona & Mendrisio & $\mathrm{TI}$ & A & $S$ & $\begin{array}{l}\text { Stagnation } 1930 / 1941 \\
+1 \text { Person } 1880 / 1888\end{array}$ \\
\hline & Gilly & Rolle & VD & ML & W & Stagnation $1880 / 1888$ \\
\hline & Bretzwil & Waldenburg & $\mathrm{BL}$ & $\mathrm{J}$ & W & +1 Person $1941 / 1950$ \\
\hline
\end{tabular}

90 Jahre

\begin{tabular}{|c|c|c|c|c|c|c|}
\hline \multirow[t]{4}{*}{$1850-1941$} & Riein & Glenner, Kreis Ilanz & GR & A & $\mathrm{E}$ & \\
\hline & Mönthal ${ }^{3}$ & Brugg & $\mathrm{AG}$ & $\mathbf{J}$ & $\mathrm{E}$ & \\
\hline & La Chaux & Cossonay & VD & ML & $\mathrm{W}$ & \\
\hline & Aurigeno & Vallemaggia & $\mathrm{TI}$ & A & $S$ & $\begin{array}{l}\text { Stagnation } 1880 / 1900 \\
+11910 / 1920\end{array}$ \\
\hline \multirow{14}{*}{$\begin{array}{l}1860-1950 \\
1870-1960\end{array}$} & Etzelkofen & Fraubrunnen & $\mathrm{BE}$ & $\mathrm{ML}$ & W & Stagnation $1880 / 1888$ \\
\hline & - & & & & & $1900 / 1910$ u. $1920 / 1930$ \\
\hline & Wasterkingen & Bülach & $\mathrm{ZH}$ & ML & $\mathrm{E}$ & \\
\hline & Nods & La Neuveville & $\mathrm{BE}$ & $\mathrm{J}$ & W & \\
\hline & San Antonio & Bellinzona & $\mathrm{TI}$ & A & $S$ & \\
\hline & Indemini ${ }^{1}$ & Locarno & TI & A & $S$ & \\
\hline & Campo-Vallemaggia 3 & Vallemaggia & TI & A & $\mathrm{S}$ & \\
\hline & Froideville & Echallens & VD & ML & $\mathrm{W}$ & \\
\hline & Cronay & Yverdon & VD & ML & W & \\
\hline & Sobrio $^{1}$ & Leventina & $\mathrm{TI}$ & A & $S$ & Stagnation $1930 / 1941$ \\
\hline & Vérossaz & St-Maurice & VS & A & $\mathrm{W}$ & Stagnation $1930 / 1941$ \\
\hline & Calonico 1 & Leventina & $\mathrm{TI}$ & A & S & +1 Person $1880 / 1888$ \\
\hline & Caviano & Locarno & $\mathrm{TI}$ & A & $\mathrm{S}$ & +1 Person $1941 / 1950$ \\
\hline & Villarzel & Payerne & VD & ML & W & +1 Person $1880 / 1888$ \\
\hline \multicolumn{7}{|l|}{$100 \mathrm{Jahre}$} \\
\hline \multirow[t]{2}{*}{$1850-1950$} & Hemberg & Neutoggenburg & SG & A & $\mathrm{E}$ & \\
\hline & Mandach & Brugg & $\mathrm{AG}$ & $\mathbf{J}$ & $\mathrm{E}$ & + 1 Person $1910 / 1920$ \\
\hline \multirow[t]{2}{*}{$1860-1960$} & Berzona 1 & Locarno & $\mathrm{TI}$ & A & $S$ & \\
\hline & Mergoscia $^{1}$ & Locarno & $\mathrm{TI}$ & A & $S$ & \\
\hline \multicolumn{7}{|l|}{110 Jahre } \\
\hline \multirow[t]{5}{*}{$1850-1960$} & Osterfingen & Oberklettgau & $\mathrm{SH}$ & $\mathbf{J}$ & $\mathrm{E}$ & \\
\hline & Thalheim & Brugg & $A G$ & $\mathrm{~J}$ & $\mathrm{E}$ & Stagnation $1930 / 1941$ \\
\hline & Niederneunforn & Frauenfeld & TG & $\mathrm{ML}$ & $\mathrm{E}$ & Stagnation $1941 / 1950$ \\
\hline & Campo-Vallemaggia ${ }^{1}, 3$ & Vallemaggia & TI & A & $\bar{S}$ & Stagnation $1860 / 1870$ \\
\hline & Mönthal ${ }^{3}$ & Brugg & $\mathrm{AG}$ & $\mathrm{J}$ & $\mathrm{E}$ & +1 Person $1941 / 1950$ \\
\hline
\end{tabular}


Die einzige Gemeinde der Schweiz, in welcher die Bevölkerungszahl in jeder Volkszählung kleiner war als in der vergangenen, ist Osterfingen im Klettgau. Von 1960 bis Ende 1967 ging ihre Einwohnerzahl weiter zurück, von 305 auf 292 Einwohner, so daß auch für 1970 eine geringere Zahl als 1960 zu erwarten ist. In drei weiteren Gemeinden lag bei allgemeiner Abnahme über alle 110 Jahre einmal Stagnation und in einer Zunahme um eine Person vor.

In einem dieser Fälle, Niederneunforn, geht dies nicht aus den Volkszählungen hervor, weil im Kanton Thurgau mehrere Gemeinden eine Munizipalgemeinde bilden und anläßlich der Volkszählungen von 1850 und 1860 nur die Zahlen für solche Gemeinwesen publiziert wurden. Nachfrage beim Statistischen Amt in Bern ergaben für die genannten Jahre 414 und 401 Einwohner in Niederneunforn.

\section{Auswirkungen in den Landschaften}

Wie bereits erwähnt, häufen sich die genannten Entvölkerungsgemeinden im Tessin, im Jura und im westlichen Mittelland. Ihre Zugehörigkeit ist in Tabelle 3 dargestellt.

In der ersten Landschaft ist der Fremdenverkehr bedeutend, aber nur in ihren verkehrsgünstig liegenden Teilen. Er wirkt jedoch, wie noch zu zeigen ist, auch auf die angrenzenden Entvölkerungstäler ein, weshalb der Landschaftstyp als Fremdenverkehrslandschaft ausgeschieden sei. Im zweiten Gebiet, dem Jura, hat sich manchenorts in waldiger Region Industrie in ursprünglicher Abhängigkeit von Wasserkraft und nicht immer an guter Verkehrslage entwickelt. Dieser Typ sei als waldreiche Industrielandschaft bezeichnet; zu ihm fallen außerhalb des Jura unsere drei ostschweizerischen Gemeinden Sternenberg, Hemberg und Trogen der Gruppe 2, da diese Gemeinden verhältnismäßig ähnliche Be-

Tabelle 3

\begin{tabular}{lcc} 
& $\begin{array}{l}\text { Gruppe 1 } \\
\text { Extreme } \\
\text { Abnahme }\end{array}$ & $\begin{array}{l}\text { Gruppe 2 } \\
\text { Regelmäßige } \\
\text { Abnahme }\end{array}$ \\
Tessin & 20 & 14 \\
$\quad$ (Umgebung Locarno) & 12 & 8 \\
Jura & 5 & $11^{11}$ \\
Westliches Mittelland & 4 & 7 \\
Andere Gebiete & 2 & 11 \\
\hline Zusammen & 31 & 43
\end{tabular}

1 Einschließlich dreier Gemeinden am Ostschweizer Voralpenrand unter ähnlichen Bedingungen. dingungen aufweisen wie solche im Jura. Das dritte relativ schwach industrialisierte Gebiet sei als landwirtschaftliches Mittelland charakterisiert.

Die Auswirkungen der Bevölkerungsabnahme in den einzelnen Landschaften sind statistisch wenig erfaßt. Anhand gewisser Unterschiede, besonders in der Physiognomie verschiedener Teilgebiete besonders beim ersten Landschaftstyp, lassen sich jedoch Rückschlüsse ziehen. Vorzüge und Mängel desjenigen Teiles des Tessins, der Fremdenverkehr aufweist, dürften hinreichend bekannt sein. Im vorliegenden Falle befinden sich Täier mit unbedeutendem Fremdenverkehr nur wenige Kilometer von dessen Zentren entfernt, sind aber topographisch klar von diesen abgetrennt, was auch die Ursache für ihre ungünstigere Verkehrslage ist.

Wohl haben diese Täler ihren früheren Charakter in wesentlich stärkerem Maße erhalten als die angrenzende Fremdenverkehrszone. Deren Nähe bildet jedoch einen Anreiz zum Verlassen der Täler. Überalterung der Bevölkerung ist eine der Folgen. Ein besonderes Problem ist jedoch, daß der Fremdenverkehr nicht nur in seinem eigentlichen Gebiet bestimmte Formen des menschlichen Zusammenlebens in Frage stellt, die als typisch für das Tessin gelten, sondern indirekt über den Bevölkerungsrückgang auch in den ablingenden Tälern.

Physiognomisch wirksamere Folgen der Entwicklung sind jedoch aufgegebene Siedlungen mit zerfallenden Gebäuden, ferner vereinzelte Neubauten, deren Stil mit dem traditionellen nicht immer in vorteilhafter Weise kontrastiert, und vor allem Veränderungen in der Vegetation. Diese war fast überall vom Menschen beeinflußt, besonders dort, wo der frühere Wald beseitigt worden war. Auf nicht mehr benötigten Wiesen und Weiden entwickelt sich meist wieder Wald, und in nicht mehr genutzten Wäldern setzen sich mit der Zeit die ursprünglichen Pflanzengesellschaften durch. Zusammenhänge dieses Vorganges mit Lokalklima und Relief sind zwar wenig quantitativ erfaßt, von der Hand zu weisen sind sie jedoch nicht. Als Hauptauswirkung des Bevölkerungsrückganges in Tessiner Tälern kann somit die Entwicklung in Richtung zum natürlichen Landschaftszustand angesehen werden.

Abgeschwächt trifft dies auch für höherliegende Teile von Landschaften des zweiten Typs. Vollständige Aufgabe bisher genutzter Flächen ist allerdings selten, häufiger ist Aufforstung bisheriger Weiden oder auch Wiesen. Wegen der Waldnutzung entstehen dabei zwar nicht die natürlichen Pflanzengesellschaften. Für die Landschaftsphysiognomie bedeutet der Vorgang immerhin eine gewisse Rückkehr zum früheren Zustand der Waldlandschaft. Gleichzeitig wächst jedoch die Bevölkerung in Talgebieten weiter. Der Typ der waldreichen Industrielandschaft bleibt damit erhalten, der Gegensatz innerhalb der Landschaft jedoch akzentuiert. 
Im vorwiegend landwirtschaftlichen, westlichen Mittelland sind die Distanzen bis zu Wachstumszentren meist größer. Eine Rückentwicklung, wie sie gezeigt wurde, läßt sich kaum feststellen. Die Entvölkerung kommt hauptsächlich als Ursache für die Erhaltung der bisherigen, vorwiegend landwirtschaftlichen Struktur in Frage. Mangels geeigneter Lehrstellen erlernt ein Teil der jungen Bevölkerung ihren Beruf anderswo und übt ihn meist auch dort aus. Es ist seltener, daß ein Rückwanderer in seinem Heimatgebiet einen für dieses neuen Erwerbszweig einführt, die bisherige Wirtschaftsstruktur bleibt somit erhalten. Wie weit Rationalisierung der Landwirtschaft als weitere Ursache dafür und wie weit als Folge des Bevölkerungsrückganges anzusehen ist, bleibt abzuklären.

Insgesamt bleibt die Landwirtschaft beim dritten Landschaftstyp jedenfalls bestehen, während sie in den anderen beiden Fällen zurückgeht. In näherer Zukunft mag ihr Anteil da und dort immerhin abnehmen, wenn in einem Gebiet von außen her systematische Industrialisierung einsetzen sollte. Da das westliche Mittelland jedoch als ganzes für die Land- wirtschaft geeignet ist, dürfte diese ihre Bedeutung insgesamt behalten.

\section{Zusammenfassung}

Außergewöhnliche Bevölkerungsverluste wurden in den meisten Fällen durch kurzfristige, gegenläufige Entwicklung unterbrochen. Deshalb sind Gemeinden mit großer Totalabnahme und gleichförmigem Rückgang meist nicht identisch. Ersteres ist besonders typisch für fremdenverkehrsarme Gebiete im Tessin, regelmäßige Abnahme ist in anderen Gebieten relativ häufiger.

Außergewöhnlich hohe Bevölkerungsverluste sind besonders typisch für Gebiete, die an ausgesprochene Wachstumszonen angrenzen, wie im Tessin und stellenweise im Jura, ununterbrochener Rückgang kommt auch in anderen Landschaften ziemlich häufig vor.

Die Gegensätze der Entwicklung zwischen einem Wachstums- und einem Entvölkerungsgebiet sind nicht nur relativ zu deren Abstand, sondern auch absolut am größten, wenn dieser Abstand gering ist. 